\title{
Report of Combined Therapy in Leprosy with Rifampicin and Isoprodian Conducted at the Bisidimo-Center, Ethiopia*
}

\author{
R. ROHDE \\ Forschungsinstitut Borstel, 2061 Borstel, West Germany
}

\begin{abstract}
On the basis of experimental results by the Borstel Research Institute, 62 patients (BL and LL cases) from the Bisidimo-Center, Ethiopia, received a combination of rifampicin and Isoprodian for a definite length of time under control and clinical conditions. In the same way 18 patients under dapsone monotherapy could be observed.

During treatment good improvement could be seen under both medications, however, the improvement was better under the combined therapy. Side-effects were exceptional; reactions occurred in both groups.

After therapy had to be discontinued, regular controls of the patients were arranged. In the dapsone group a deterioration was soon found, and treatment had to be continued; After combined therapy, however, the trend of improvement continued, the clinical and bacteriological improvement has shown to be progressive; up to now (in many cases more than 2 years after treatment was stopped) no persistent signs of relapse could be found. The patients will continue under further observation.
\end{abstract}

On the basis of experimental results by the Borstel Research Institute and after first clinical experience was available, 62 leprosy patients from the BisidimoCenter, Ethiopia, were given an antimycobacterial combined therapy for a definite length of time.

The combined therapy consisted of rifampicin and Isoprodian. The latter is a ready-made combination of prothionamide, dapsone and isoniazid. Before Isoprodian was available, the combination partners of rifampicin were given singly and instead of dapsone a trimethoprim-sulphonamide preparation was used in the combination. The patients all suffered from the lepromatous type of leprosy (BL and LL cases), the Bacteriological Index was highly positive. Most of the patients were new cases, a smaller group had received dapsone therapy previously however, with unsatisfying results. During the time of therapy the patients were admitted to the hospital and the medication was given daily under personal control, rifampicin $10 \mathrm{mg} / \mathrm{kg}$ body weight, Isoprodian 2-4 tablets according to body weight. Under the same conditions a group of 18 control patients was under observation, receiving dapsone therapy in a high daily dosage-11 patients received $25 \mathrm{mg}, 7$ patients $100 \mathrm{mg}$ dapsone daily. For bacteriological diagnosis

\footnotetext{
* This investigation received financial support from the German Leprosy Relief Association.
} 
skin smears were done fortnightly and biopsies were taken monthly. Besides the routine laboratory examinations a photographic documentation was undertaken.

The duration of stay in the hospital and consequently the time of treatment was limited to $1 / 4$ or $1 / 2$ year respectively as the patients wanted to return to their villages and refused to stay any longer in the Center. However, further control was possible, their clinical and bacteriological status was regularly registered and documented by photography. The cases will also be followed-up in the future.

\section{Clinical Results}

During the time of treatment an obvious improvement could be seen in both groups (combined therapy and dapsone monotherapy). Those patients, however, that had improved most, could be found in the combined therapy group. Reactions were present in both groups, in more severe cases thalidomide was given with remarkably good results, cortisones were not used, the therapy was not interrupted, and the medication was not reduced in dosage. The preliminary high values for leucocyte count and ESR turned to normal under treatment, but in the combined therapy group this was more evident and quicker. With the exception of 4 cases who became infected during a viruș-hepatitis epidemic ( 2 patients of the dapsone group and 2 patients of the combined therapy group) in no case could an impairment of the liver-function be found, there were as well no signs of a toxic anaemia and no impairment of the kidney function. The drugs were very well tolerated, and side effects were exceptional. Under rifampicin and Isoprodian $70 \%$ of the patients were without any complaints during 3 months' treatment.

After therapy was discontinued it could be noticed during the continued follow-up that those patients who had received the combined therapy showed a progressive clinical improvement; they were without complaints, the aspect of the disease showed a step by step improvement up to the present. In many cases a period of 2 years after the initial treatment can be surveyed already. A quite different situation can be seen in the group that had received the dapsone monotherapy; after a short period of improvement in most cases a deterioration followed that made continuation of treatment necessary.

\section{Bacteriological Results}

The bacteriological results during the time of therapy and the period after therapy correspond with the results of the clinical observations. During the therapy a slow decrease in the Bacteriological Index took place. The rate of decrease was better under combined therapy than under dapsone monotherapy. The Morphological Index became negative in most patients during the first weeks, the most rapid decrease again being observed under combined therapy.

In the period of follow-up after the course of therapy it was found that in those patients who had received the combined therapy a further and progressive decrease of the Bacteriological Index was evident; 1 year after therapy about 50\% of the patients became bacteriologically negative. In the dapsone group a different situation was found. In most patients a deterioration of the bacteriological findings followed an initial improvement the Bacteriological Index showed an increase and the Morphological Index became positive again.

As example of these bacteriological and clinical aspects results in 2 smaller therapy groups are shown on the following 2 figures. The photographs document the outstanding clinical improvement of leprosy patients after a short-term treatment with the combined therapy. 
Figs 1 and 2 show the comparison of 2 groups with the longest survey-time after therapy. Group 1: 18 patients, dapsone monotherapy $(0.5$ or $2 \mathrm{mg} / \mathrm{kg}), 3$ months.

Group 2: 16 patients, combined therapy, 3 months, rifampicin $(10 \mathrm{mg} / \mathrm{kg})$, Ethionamide $(10 \mathrm{mg} / \mathrm{kg})$, isoniazid $(5 \mathrm{mg} / \mathrm{kg})$, Trimethoprim-Sulphonamide $(20 \mathrm{mg} / \mathrm{kg})$.

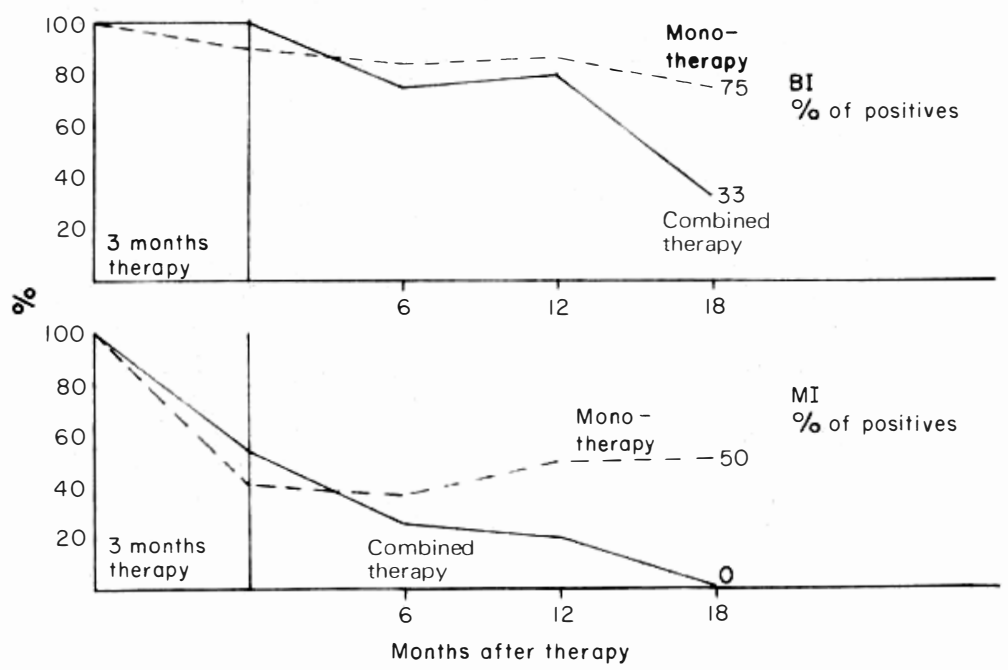

Fig. 1. Bacteriological results. The percentage of BI and MI positive cases show initially the same course, $1 / 2$ and 1 year respectively after therapy a change occurs. After combined therapy the initial trend continues through the time without treatment in direction of bacterial negativity, In opposite the trend after dapsone monotherapy changes and leads back again to the condition of origin; this corresponds with the observation of the clinical appearance.

Months after therapy
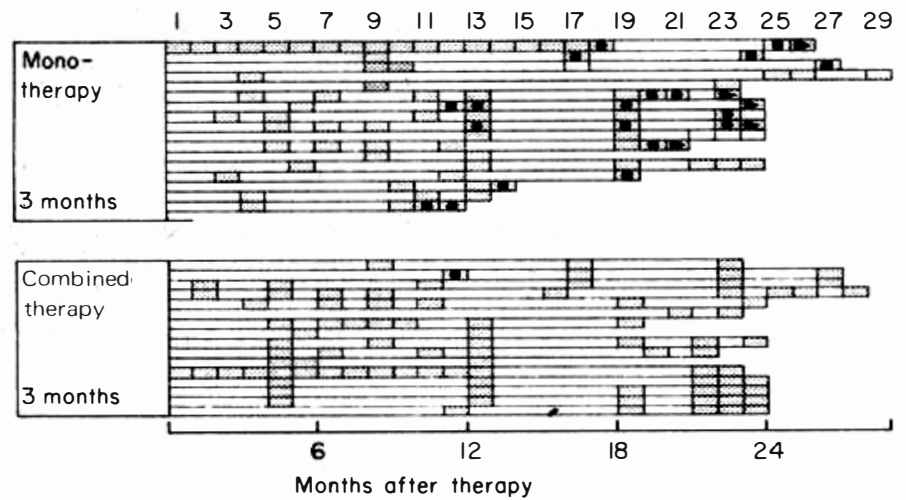

Fig. 2. Clinical symptoms. The reappearance of new and persistent symptoms (lepromata and patches) after therapy was discontinued was only noticed af ter short dapsone monotherapy. In these patients treatment was started again. Such new persistent symptoms were not found until now after 3 months' combined therapy, not even in those patients who were treated before unsuccessfully for many years with monotherapy.

Each line shows the time after therapy of each patient. Each grey field is a visit to this patient and a control of his clinical and bacteriological status.

$(\bullet)$ the appearance of new lepromata or patches; $(\triangleright)$ shows that the patient is under treatment again. 


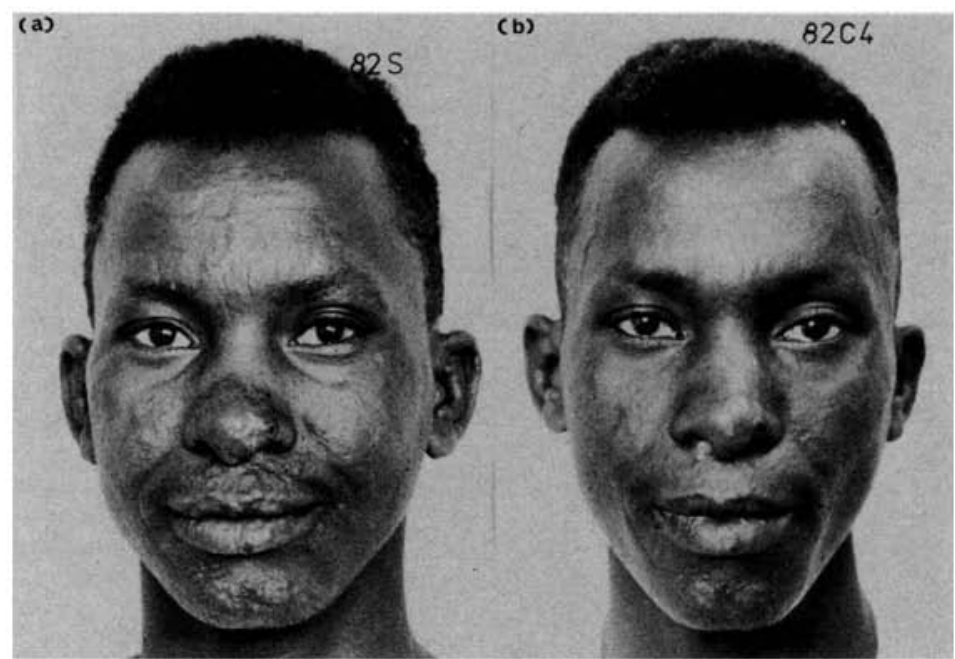

Fig. 3. Patient No. 82, not pretreated. (a) Before treatment. (b) After 3 months' combined therapy and a 4 months' period without further treatment.

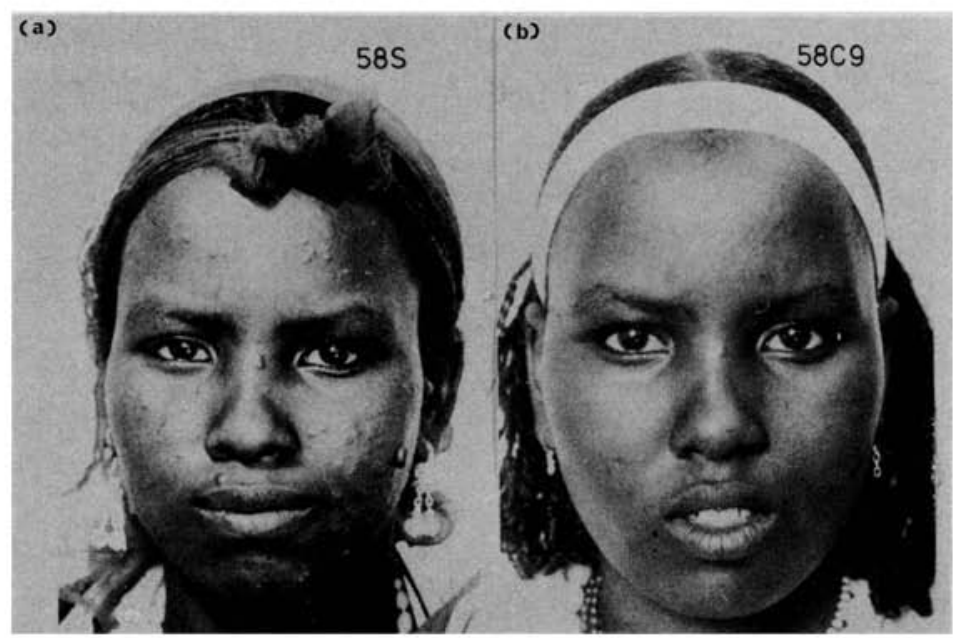

Fig. 4. Patient No. 58, not pretreated. (a) Before treatment BI $1.1 \times 10^{6}$ bacteria/mg skin biopsy MI 7.7\%. (b) After a 7 months' combined therapy and a 9 months' period without further treatment. BI negative. 


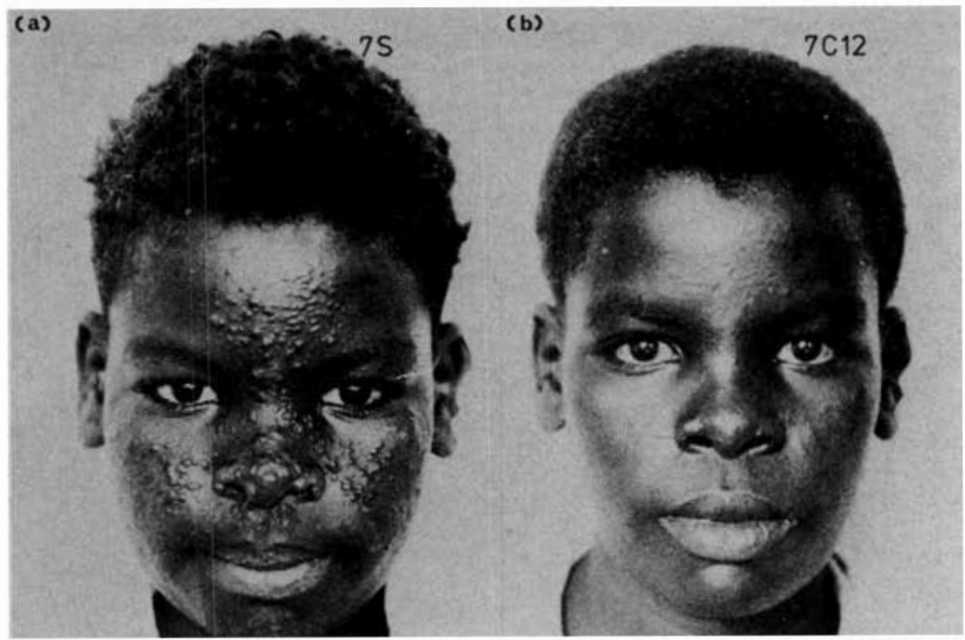

Fig. 5. Patient No. 7, not pretreated. (a) before treatment. BI $5.6 \times 10^{5}$ bacteria/mg skin biopsy. (b) After a 7 months' combined therapy and a 12 months' period without further treatment. BI negative.

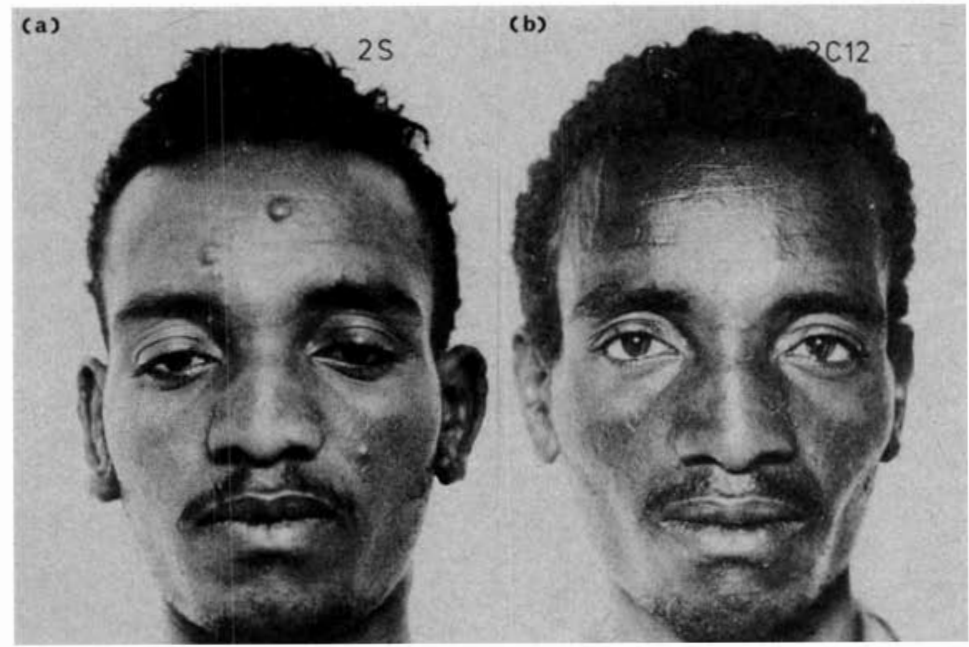

Fig. 6. Patient No. 2, not pretreated. (a) Before treatment BI $1.7 \times 10^{4}$ bacteria/mg skin biopsy. (b) After a 7 months' combined therapy and a 12 months' period without further treatment. BI negative. 


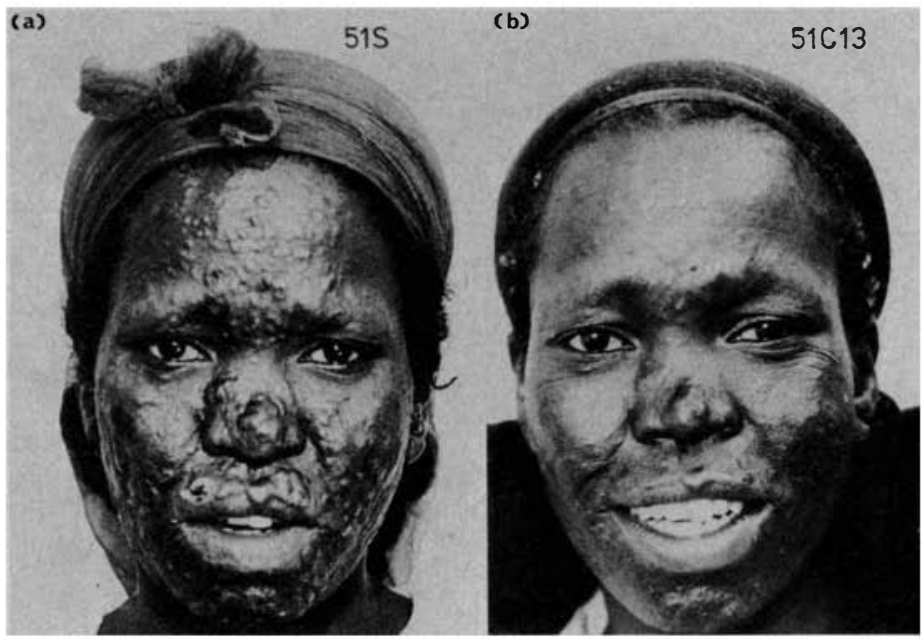

Fig. 7. Patient No. 51, regularly pretreated with dapsone and Ciba 1906 for 10 years. (a) Before treatment, BI $1.1 \times 10^{6}$ bacteria/mg skin biopsy, MI $11 \%$. (b) After 3 months' combined therapy and 13 months' period without further treatment. BI $5.1 \times 10^{3}$, MI negative.

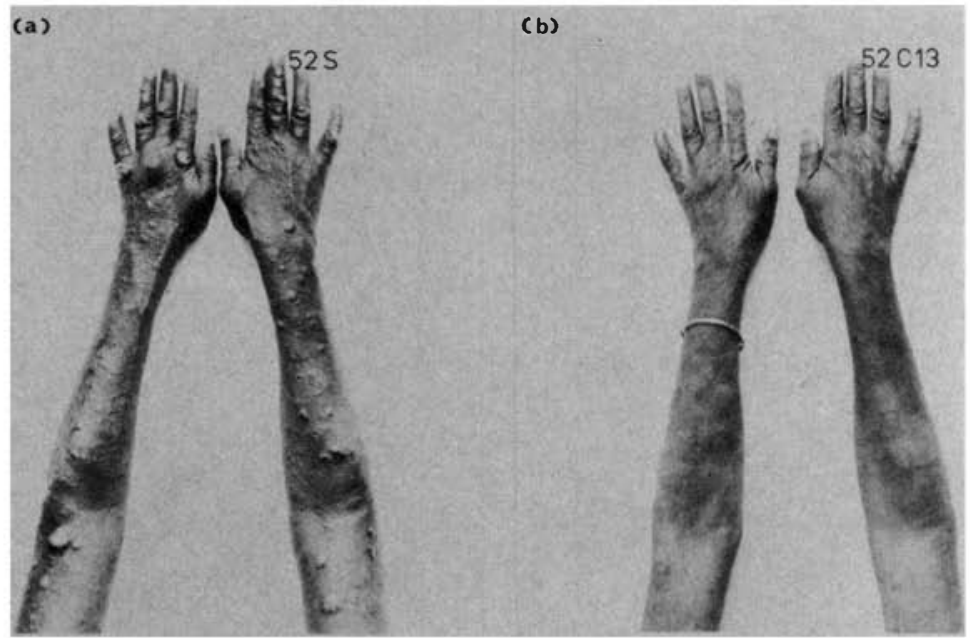

Fig. 8. Patient No. 52, regularly pretreated with dapsone and Ciba 1906 for 9 years. (a) Before treatment. (b) After 3 months' combined therapy and 13 months' period without further treatment. 


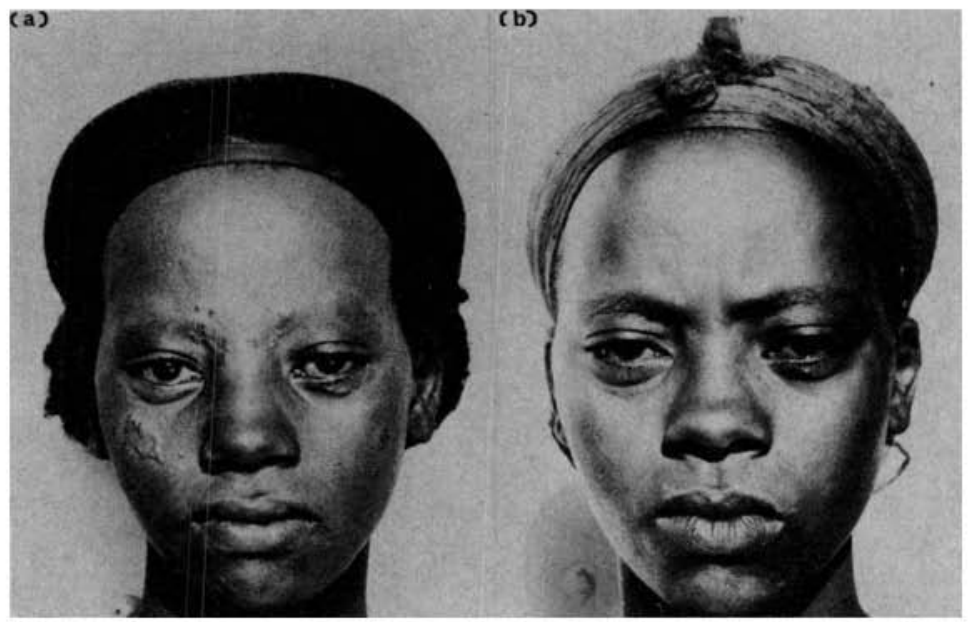

Fig. 9. Patient No. 56, not pretreated. (a) Before treatment. (b) After 3 months' combined therapy and 12 months' period without further treatment. The regrowth of eyebrows is remarkable. 